\title{
Article
}

\section{1-Hypergroups of Small Sizes}

\author{
Mario De Salvo ${ }^{1, *(\mathbb{D})}$, Dario Fasino ${ }^{2}\left(\mathbb{D}\right.$, Domenico Freni ${ }^{2}\left(\mathbb{D}\right.$ and Giovanni Lo Faro ${ }^{1}(\mathbb{D})$ \\ 1 Dipartimento di Scienze Matematiche e Informatiche, Scienze Fisiche e Scienze della Terra, \\ Università di Messina, 98122 Messina, Italy; lofaro@unime.it \\ 2 Dipartimento di Scienze Matematiche, Informatiche e Fisiche, Università di Udine, 33100 Udine, Italy; \\ dario.fasino@uniud.it (D.F.); domenico.freni@uniud.it (D.F.) \\ * Correspondence: desalvo@unime.it
}

Citation: De Salvo, M.; Fasino, D.; Freni, D.; Lo Faro, G. 1-Hypergroups of Small Sizes. Mathematics 2021, 9 , 108. https://doi.org/10.3390/ math9020108

Received: 7 December 2020 Accepted: 2 January 2021 Published: 6 January 2021

Publisher's Note: MDPI stays neutral with regard to jurisdictional clai$\mathrm{ms}$ in published maps and institutional affiliations.

Copyright: (C) 2021 by the authors. Licensee MDPI, Basel, Switzerland. This article is an open access article distributed under the terms and conditions of the Creative Commons Attribution (CC BY) license (https:// creativecommons.org/licenses/by/ $4.0 /)$.

\begin{abstract}
In this paper, we show a new construction of hypergroups that, under appropriate conditions, are complete hypergroups or non-complete 1-hypergroups. Furthermore, we classify the 1-hypergroups of size 5 and 6 based on the partition induced by the fundamental relation $\beta$. Many of these hypergroups can be obtained using the aforesaid hypergroup construction.
\end{abstract}

Keywords: hypergroups; complete hypergroups; fundamental relations

\section{Introduction}

Hypercompositional algebra is a branch of Algebra experiencing a surge of activity nowadays that concerns the study of hyperstructures, that is, algebraic structures where the composition of two elements is a set rather than a single element [1]. The subjects, methods, and goals of the hypercompositional algebra are very different from those of classic algebra. However, the two fields are connected by certain equivalence relations, called fundamental relations [2,3]. Through fundamental relations, the analysis of algebraic hyperstructures can make use of the wealth of tools typical of classical algebra. Indeed, fundamental relations are peculiar equivalence relations defined on hyperstructures, in such a way that the associated quotient set is one of the classical algebraic structures.

More precisely, a fundamental relation is the smallest equivalence relation defined on the support of a hyperstructure such that the corresponding quotient set is a classical structure having operational properties analogous to those of the hyperstructure [4-7]. For example, the quotient structure modulo the equivalence $\beta^{*}$ defined on a semihypergroup (or a hypergroup) is a semigroup (or a group, respectively) [2,8-10]. Analogous definitions and results are also known in hyperstructures endowed with more than one operation, see e.g., [11]. Moreover, hypergroups can be classified according to the height of a $\beta^{*}$-class, that is, the least number of order-2 hyperproducts that can cover that class, see [12].

If $(H, \circ)$ is a hypergroup and $\varphi: H \mapsto H / \beta^{*}$ is the canonical projection then the kernel $\omega_{H}=\varphi^{-1}\left(1_{H / \beta^{*}}\right)$ is the heart of $(H, \circ)$. The heart of a hypergroup plays a very important role in hypergroup theory. Indeed, if we know the structure of $\omega_{H}$ then we have detailed information on the partition determined by relation $\beta^{*}$ since $\beta^{*}(x)=\omega_{H} \circ x=x \circ \omega_{H}$, for all $x \in H$. When the heart of a hypergroup $(H, 0)$ has only one element $\varepsilon$, this element is also the identity of $(H, \circ)$, since $x \in \beta^{*}(x)=x \circ \varepsilon=\varepsilon \circ x$. According to a definition introduced by Corsini in [4], the hypergroups whose heart has size 1 are called 1-hypergroups. In ([12] Theorem 2), we characterized the 1-hypergroups in terms of the height of their heart, and in [13] Sadrabadi and Davvaz investigated sequences of join spaces associated with non-complete 1-hypergroups.

In this paper, we deepen the knowledge of 1-hypergroups. In particular, we classify the 1-hypergroups of cardinalities up to 6 on the basis of the partition of $H$ induced by $\beta^{*}$. This technique allows us to explicitly construct all 1-hypergroups of order 5 , and enumerate those of order 6 by means of scientific computing software. We recall that the study of small-size algebraic hyperstructures is both a practical tool to analyze more 
elaborate structures and a well-established research topic in itself. In fact, the enumeration and classification of hyperstructures having small cardinality have made it possible to solve various relevant existence issues in hyperstructure theory, see e.g., [14-17].

The plan of this paper is the following: In the forthcoming Section 2, we introduce the basic definitions, notations, and fundamental facts to be used throughout the paper. In Section 3, we present a new construction of hypergroups that, under appropriate hypotheses, are complete hypergroups or non-complete 1-hypergroups. Moreover, we prove a few results concerning the $\beta$-classes of 1-hypergroups and sufficient conditions for 1hypergroups to be complete, which are relevant in subsequent sections. In Section 4, we determine the 1-hypergroups of size 5, up to isomorphisms. In Section 5 we classify the 1-hypergroups of size 6 , up to isomorphisms. The 1-hypergroups of size 4, and many 1-hypergroups of size 5 and 6, can be determined by the construction defined in Section 3 . The paper ends with some conclusions and directions for future research in Section 6.

\section{Basic Definitions and Results}

Let $H$ be a non-empty set and let $\mathcal{P}^{*}(H)$ be the set of all non-empty subsets of $H$. A hyperproduct $\circ$ on $H$ is a map from $H \times H$ to $\mathcal{P}^{*}(H)$. For all $x, y \in H$, the subset $x \circ y$ is the hyperproduct of $x$ and $y$. If $A, B$ are non-empty subsets of $H$ then $A \circ B=\bigcup_{x \in A, y \in B} x \circ y$.

A semihypergroup is a non-empty set $H$ endowed with an associative hyperproduct $\circ$, that is, $(x \circ y) \circ z=x \circ(y \circ z)$ for all $x, y, z \in H$. We say that a semihypergroup $(H, \circ)$ is a hypergroup if for all $x \in H$, we have $x \circ H=H \circ x=H$, the so-called reproducibility property.

A non-empty subset $K$ of a semihypergroup $(H, \circ)$ is called a subsemihypergroup of $(H, \circ)$ if it is closed with respect to the hyperproduct $\circ$, that is, $x \circ y \subseteq K$ for all $x, y \in K$. A non-empty subset $K$ of a hypergroup $(H, 0)$ is called a subhypergroup of $(H, 0)$ if $x \circ K=K \circ x=K$, for all $x \in K$. If a subhypergroup is isomorphic to a group, then we say that it is a subgroup of $(H, 0)$.

Given a semihypergroup $(H, 0)$, the relation $\beta^{*}$ in $H$ is the transitive closure of the relation $\beta=\bigcup_{n \geq 1} \beta_{n}$ where $\beta_{1}$ is the diagonal relation in $H$ and, for every integer $n>1$, $\beta_{n}$ is defined recursively as follows:

$$
x \beta_{n} y \Longleftrightarrow \exists\left(z_{1}, \ldots, z_{n}\right) \in H^{n}:\{x, y\} \subseteq z_{1} \circ z_{2} \circ \cdots \circ z_{n} .
$$

We let $\beta^{*}(x)$ denote the $\beta^{*}$-class of $x \in H$. The relations $\beta$ and $\beta^{*}$ are among the best known fundamental relations [3]. Their relevance in hyperstructure theory stems from the following facts [2]: If $(H, 0)$ is a semihypergroup (respectively, a hypergroup) then the quotient set $H / \beta^{*}$ equipped with the operation $\beta^{*}(x) \otimes \beta^{*}(y)=\beta^{*}(z)$ for all $x, y \in H$ and $z \in x \circ y$ is a semigroup (respectively, a group). Moreover, the relation $\beta^{*}$ is the smallest strongly regular equivalence on $H$ such that the quotient $H / \beta^{*}$ is a semigroup (resp., a group). The canonical projection $\varphi: H \mapsto H / \beta^{*}$ is a good homomorphism, that is, $\varphi(x \circ y)=\varphi(x) \otimes \varphi(y)$ for all $x, y \in H$. The relations $\beta$ and $\beta^{*}$ are also useful to introduce notable families of semihypergroups and hypergroups, including the fully simple semihypergroups [18-20] and the 0-simple semihypergroups [14,21-23], having interesting connections with partially ordered sets and integer sequences. Furthermore, we recall from $[8,10]$ that if $(H, \circ)$ is a hypergroup then $\beta$ is transitive, so that $\beta=\beta^{*}$ in every hypergroup.

If $(H, \circ)$ is a hypergroup then $H / \beta^{*}$ is a group and the kernel $\omega_{H}=\varphi^{-1}\left(1_{H / \beta^{*}}\right)$ of $\varphi$ is the heart of $(H, \circ)$. Furthermore, if $\left|\omega_{H}\right|=1$ then $(H, \circ)$ is a 1-hypergroup. For later reference, we collect in the following theorem a couple of classic results concerning the heart of a hypergroup, see [2,4].

Theorem 1. Let $(H, \circ)$ be a hypergroup. Then,

1. $\beta(x)=x \circ \omega_{H}=\omega_{H} \circ x$, for all $x \in H$;

2. $(x \circ y) \cap \omega_{H} \neq \varnothing \Longleftrightarrow(y \circ x) \cap \omega_{H} \neq \varnothing$, for all $x, y \in H$. 
If $A$ is a non-empty set of a semihypergroup $(H, \circ)$ then we say that $A$ is a complete part if it fulfills the following condition: for every $n \in \mathbb{N}-\{0\}$ and $\left(x_{1}, x_{2}, \ldots, x_{n}\right) \in H^{n}$,

$$
\left(x_{1} \circ \cdots \circ x_{n}\right) \cap A \neq \varnothing \Longrightarrow\left(x_{1} \circ \cdots \circ x_{n}\right) \subseteq A .
$$

For every non-empty set $X$ of $H$, the intersection of all the complete parts containing $X$ is called the complete closure of $X$ and is denoted with $\mathfrak{C}(X)$. Clearly, $X$ is a complete part of $(H, \circ)$ if and only if $\mathfrak{C}(X)=X$. If $(H, \circ)$ is a semihypergroup and $\varphi: H \mapsto H / \beta^{*}$ is the canonical projection then, for all non-empty set $A \subseteq H$, we have $\mathfrak{C}(A)=\varphi^{-1}(\varphi(A))$. Moreover, if $(H, 0)$ is a hypergroup then

$$
\mathfrak{C}(A)=\varphi^{-1}(\varphi(A))=A \circ \omega_{H}=\omega_{H} \circ A .
$$

A semihypergroup or hypergroup $(H, \circ)$ is complete if $x \circ y=\mathfrak{C}(x \circ y)$ for all $x, y \in H$. If $(H, \circ)$ is a complete (semi-)hypergroup then

$$
x \circ y=\mathfrak{C}(a)=\beta^{*}(a),
$$

for every $x, y \in H$ and $a \in x \circ y$. Recently, Sonea and Cristea analyzed in [24] the commutativity degree of complete hypergroups, stressing their similarities and differences with respect to group theory. The interested reader can find all relevant definitions, properties and applications of hyperstructures and fundamental relations, even in more abstract contexts, also in [4,25-30].

In what follows, if $(H, \circ)$ is a finite hypergroup and $|H|=n$ then we set $H=\{1,2, \ldots, n\}$. Moreover, if $(H, O)$ is a (possibly infinite) 1-hypergroup then we adopt the convention $\omega_{H}=\{1\}$.

\section{Main Results}

In this section, we prove some results which will be used to classify the 1-hypergroups of sizes 4,5 and 6 . To this aim, we now give a construction of hypergroups which, under certain conditions, allows us to determine non-complete 1-hypergroups, starting from complete 1-hypergroups.

\subsection{A New Construction}

Let $(G, \cdot)$ be a group with $|G| \geq 2$ and let $\mathfrak{F}=\left\{A_{k}\right\}_{k \in G}$ be a family of non-empty and pairwise disjoint sets indexed by $G$. Let $i, j \in G-\left\{1_{G}\right\}$ be not necessarily distinct elements and let $\varphi: A_{i} \times A_{j} \mapsto \mathcal{P}^{*}\left(A_{i j}\right)$ be any function such that for all $a \in A_{i}$ and $b \in A_{j}$

$$
\bigcup_{x \in A_{j}} \varphi(a, x)=\bigcup_{x \in A_{i}} \varphi(x, b)=A_{i j} .
$$

As a shorthand, introduce the infix notation $\star: A_{i} \times A_{j} \mapsto A_{i j}$ defined by $a \star b=$ $\varphi(a, b)$ for every $a \in A_{i}$ and $b \in A_{j}$. This operation is naturally extended to sets as follows: for $X \in \mathcal{P}^{*}\left(A_{i}\right)$ and $Y \in \mathcal{P}^{*}\left(A_{j}\right)$ let

$$
a \star Y=\bigcup_{y \in Y} a \star y, \quad X \star b=\bigcup_{x \in X}=x \star b, \quad X \star Y=\bigcup_{x \in X, y \in Y} x \star y .
$$

Hence, the condition (1) can be reformulated as $A_{i} \star b=a \star A_{j}=A_{i j}$. Now, let $H=\bigcup_{k \in G} A_{k}$ and consider the hyperproduct $\circ: H \times H \mapsto \mathcal{P}^{*}(H)$ defined as follows: for all $x, y \in H$ let

$$
x \circ y= \begin{cases}A_{r s} & \text { if } x \in A_{r}, y \in A_{s} \text { and }(r, s) \neq(i, j), \\ x \star y & \text { if } x \in A_{i} \text { and } y \in A_{j} .\end{cases}
$$

The following result shows the usefulness of this construction. 
Proposition 1. In the previous notation,

1. for every $r, s \in G$ and $x \in A_{s}$ we have $A_{r} \circ x=A_{r s}$ and $x \circ A_{r}=A_{s r}$;

2. the hyperproduct $\circ$ is associative: for every $r, s, t \in G, x \in A_{r}, y \in A_{s}$ and $z \in A_{t}$, we have

$$
(x \circ y) \circ z=A_{(r s) t}=A_{r(s t)}=x \circ(y \circ z)
$$

3. for every $z_{1}, z_{2}, \ldots, z_{n} \in H$ with $n \geq 3$ there exists $r \in G$ such that $z_{1} \circ z_{2} \circ \cdots \circ z_{n}=A_{r}$;

4. $(H, \circ)$ is a hypergroup such that $\beta=\beta_{2}$;

5. for every $x \in H, x \in A_{k} \Longleftrightarrow \beta(x)=A_{k}$;

6. $H / \beta \cong G$ and $\omega_{H}=A_{1_{G}}$;

7. if $\left|A_{1_{G}}\right|=1$ then $(H, \circ)$ is a 1-hypergroup;

8. $(H, \circ)$ is complete if and only if $a \star b=A_{i j}$ for every $a \in A_{i}$ and $b \in A_{j}$.

Proof. In the stated hypothesis we have:

1. Let $r, s \in G$ and $x \in A_{s}$. If $r \neq i$ or $s \neq j$ then $A_{r} \circ x=\bigcup_{y \in A_{r}}(y \circ x)=A_{r s}$. Otherwise, if $r=i$ and $s=j$ then $A_{r} \circ x=A_{i} \circ x=A_{i} \star x=A_{i j}$ by Equation (1). The identity $x \circ A_{r}=A_{s r}$ can be derived by similar arguments.

2. For every $r, s, t \in G$ and $x \in A_{r}, y \in A_{s}$ and $z \in A_{t}$, we have

$$
(r, s) \neq(i, j) \Longrightarrow(x \circ y) \circ z=A_{r s} \circ z=A_{(r s) t} .
$$

Moreover, since $j \neq 1_{G}$ and the sets of the family $\mathfrak{F}$ are pairwise disjoint, if $(r, s)=(i, j)$ then $A_{i j} \neq A_{i}$ and $a \circ z=A_{(i j) t}=A_{(r s) t}$, for every $a \in x \star y \subseteq A_{i j}$. Therefore,

$$
(x \circ y) \circ z=(x \star y) \circ z=\bigcup_{a \in x \star y} a \circ z=A_{(r s) t} .
$$

The identity $x \circ(y \circ z)=A_{(r s) t}$ follows analogously.

3. It suffices to apply points 1 . and 2. above and proceed by induction on $n$.

4. By 2., $(H, 0)$ is a semihypergroup. To prove that it is a hypergroup it remains to prove that the hyperproduct $\circ$ is reproducible. Let $x \in H$. If $x \in A_{i}$ then

$$
\begin{aligned}
x \circ H=\bigcup_{y \in H} x \circ y & =\left(\bigcup_{y \in A_{j}} x \circ y\right) \bigcup\left(\bigcup_{y \in H-A_{j}} x \circ y\right) \\
& =\left(x \circ A_{j}\right) \bigcup\left(\bigcup_{r \in G-\{j\}} A_{i r}\right)=A_{i j} \cup\left(H-A_{i j}\right)=H .
\end{aligned}
$$

If $x \in A_{h}$ with $h \neq i$ then $x \circ H=\bigcup_{y \in H} x \circ y=\bigcup_{r \in G} A_{h r}=H$ because $h G=G$. Therefore $x \circ H=H$. The identity $H \circ x=H$ can be shown analogously, by considering separately the cases $x \in A_{j}$ and $x \in H-A_{j}$. Therefore $\circ$ is reproducible and $(H, \circ)$ is a hypergroup. Consequently, we have the chain of inclusions

$$
\beta_{1} \subseteq \beta_{2} \subseteq \cdots \subseteq \beta_{n} \subseteq \cdots
$$

Now, let $x, y \in H$ be such that $x \beta y$. Hence, there exists $n \geq 3$ such that $x \beta_{n} y$. By point 3., there exists $r \in G$ such that $\{x, y\} \subseteq A_{r}$. For every $a \in A_{1}$ we have $\{x, y\} \subseteq A_{r}=x \circ a$ and we obtain $x \beta_{2} y$.

5. Let $x \in A_{k}$. If $a \in A_{1}$ then $A_{k}=x \circ a$, and so $y \in A_{k}$ implies $y \beta_{2} x$. Conversely, if $y \beta_{2} x$ then there exist $a, b \in H$ such that $\{x, y\} \subseteq a \circ b$. From the definition of the hyperproduct $\circ$ it follows that there exists $r \in G$ such that $a \circ b \subseteq A_{r}$. Therefore, since $x \in A_{k} \cap A_{r}$ and the sets of the family $\mathfrak{F}$ are pairwise disjoint, we obtain $y \in a \circ b \subseteq A_{r}=A_{k}$. Finally, $A_{k}=\beta(x)$ because $\beta_{2}=\beta$.

6. The application $f: G \mapsto H / \beta$ such that $f(k)=A_{k}$ is a group isomorphism. Moreover, since $1_{H / \beta}=f\left(1_{G}\right)=A_{1_{G}}$, we conclude $\omega_{H}=A_{1_{G}}$. 
7. The claim follows immediately from points 4 . and 6 .

8. Trivial.

We stress the fact that the hypothesis $i, j \neq 1_{G}$ placed in the above construction is essential for the validity of Proposition 1. In fact, if that hypothesis is not fulfilled then the hyperproduct $\circ$ defined by our construction may not be associative, as shown by the following example.

Example 1. Let $G \cong \mathbb{Z}_{2},(i, j)=(2,1), A_{1}=\{a, b\}$, and $A_{2}=\{c, d\}$. Consider the function $\varphi: A_{2} \times A_{1} \mapsto \mathcal{P}^{*}\left(A_{2}\right)$ represented by the following table:

\begin{tabular}{lll}
\hline$\star$ & $a$ & $b$ \\
\hline$c$ & $c$ & $d$ \\
$d$ & $d$ & $c$
\end{tabular}

In this case, the previous construction determines the following hyperproduct table:

\begin{tabular}{ccccc}
\hline$\circ$ & $a$ & $b$ & $c$ & $d$ \\
\hline$a$ & $A_{1}$ & $A_{1}$ & $A_{2}$ & $A_{2}$ \\
$b$ & $A_{1}$ & $A_{1}$ & $A_{2}$ & $A_{2}$ \\
$c$ & $c$ & $d$ & $A_{1}$ & $A_{1}$ \\
$d$ & $d$ & $c$ & $A_{1}$ & $A_{1}$ \\
\hline
\end{tabular}

We have $c \star A_{1}=d \star A_{1}=A_{2}$ and $A_{2} \star a=A_{2} \star b=A_{2}$, hence the hyperproduct $\circ$ is not associative because

$$
(c \circ a) \circ a=\{c\} \subset A_{2} \quad c \circ(a \circ a)=c \circ A_{1}=A_{2} .
$$

Remark 1. The complete hypergroups have been characterized by Corsini in [4] by means of a construction very similar to ours. In fact, the above construction reduces to the one in [4] if the condition in Equation (1) is replaced by $\varphi(a, b)=A_{i j}$ for every $a \in A_{i}$ and $b \in A_{j}$. In that case, the hypergroup thus produced is complete.

\subsection{Auxiliary Results}

Now, we prove two results that are valid in every hypergroup. Recall that in every hypergroup the relation $\beta$ is an equivalence coinciding with $\beta^{*}[8,10]$.

Proposition 2. Let $(H, \circ)$ be a hypergroup. For all $x, y \in H, x \circ \beta(y)=\beta(x) \circ \beta(y)=\beta(x) \circ y$.

Proof. By Theorem 1(1) we have $x \circ \beta(y)=x \circ\left(\omega_{H} \circ \omega_{H} \circ y\right)=\left(x \circ \omega_{H}\right) \circ\left(\omega_{H} \circ y\right)=$ $\beta(x) \circ \beta(y)=\beta(x) \circ\left(\omega_{H} \circ y\right)=\left(\beta(x) \circ \omega_{H}\right) \circ y=\beta(x) \circ y$.

Proposition 3. Let $(H, \circ)$ be a hypergroup. If $a$ is an elements of $H$ such that $\beta(a)=\{a\}$ then both $a \circ b$ and $b \circ$ a are $\beta$-classes, for all $b \in H$.

Proof. By Proposition 2, $a \circ b=\beta(a) \circ b=\beta(a) \circ \beta(b)$. The identity $b \circ a=\beta(b) \circ \beta(a)$ is obtained analogously.

The next results concern the properties of 1-hypergroups.

Corollary 1. Let $(H, \circ)$ be a 1-hypergroup. If there exists only one $\beta$-class of size greater than 1 then $H$ is complete. 
Proof. Let $\beta(x)$ be the only $\beta$-class with $|\beta(x)|>1$. By Proposition 3, we only have to prove that if $a \in \beta(x)$ then both $a \circ b$ and $b \circ a$ are $\beta$-classes, for all $b \in H$. Let $\varphi: H \mapsto H / \beta$ be the canonical projection and $c \in a \circ b$. We prove that $a \circ b=\beta(c)$. If $|\beta(c)|=1$ then $a \circ b=\beta(c)$. If $|\beta(c)|>1$ then $\beta(c)=\beta(x)=\beta(a)$. Consequently,

$$
\varphi(x)=\varphi(c)=\varphi(a) \otimes \varphi(b)=\varphi(x) \otimes \varphi(b),
$$

hence $\varphi(b)=1_{H / \beta}$ and we have $b \in \omega_{H}=\{1\}$. Finally, $a \circ b=a \circ 1=\beta(a)=\beta(c)$. Analogous arguments can prove that also $b \circ a$ is a $\beta$-class.

Remark 2. If $H$ is not a complete 1-hypergroup and $H$ owns exactly two $\beta$-classes, $\beta(a)$ and $\beta(b)$, of size greater than 1 , then $\beta(a) \circ \beta(a)=\beta(b)$ or $\beta(b) \circ \beta(b)=\beta(a)$.

From Corollary 1 we get the following results.

Proposition 4. Let $(H, \circ)$ be a finite 1-hypergroup. If $|H / \beta|=p$ and there exists a $\beta$-class of size $|H|-p+1$, then $H$ is a complete hypergroup.

The previous proposition allows us to find a simple proof to a result shown in [4] providing a taxonomy of all 1-hypergroups of size up to 4 .

Theorem 2. If $(H, \circ)$ is 1-hypergroup and $|H| \leq 4$ then $(H, \circ)$ is a complete hypergroup. Moreover, $(H, \circ)$ is either a group or is one of the hypergroups described by the following three hyperproduct tables, up to isomorphisms:

\begin{tabular}{cccc}
\hline$\circ$ & 1 & 2 & 3 \\
\hline 1 & 1 & 2,3 & 2,3 \\
2 & 2,3 & 1 & 1 \\
3 & 2,3 & 1 & 1 \\
\hline
\end{tabular}

\begin{tabular}{|c|c|c|c|c|c|}
\hline 0 & \multicolumn{2}{|c|}{1} & 2 & 3 & 4 \\
\hline 1 & \multicolumn{2}{|c|}{1} & $2,3,4$ & $2,3,4$ & $2,3,4$ \\
\hline 2 & \multicolumn{2}{|c|}{$2,3,4$} & 1 & 1 & 1 \\
\hline 3 & \multicolumn{2}{|c|}{$2,3,4$} & 1 & 1 & 1 \\
\hline 4 & \multicolumn{2}{|c|}{$2,3,4$} & 1 & 1 & 1 \\
\hline & $\circ$ & 1 & 2 & 3 & 4 \\
\hline & 1 & 1 & 2,3 & 2,3 & 4 \\
\hline & 2 & 2,3 & 4 & 4 & 1 \\
\hline & 3 & 2,3 & 4 & 4 & 1 \\
\hline & 4 & 4 & 1 & 1 & 2,3 \\
\hline
\end{tabular}

Proof. Let $(H, \circ)$ be a 1-hypergroup of size $\leq 4$ that is not a group. Two cases are possible: (i) $|H|=3$ and $|H / \beta|=2$; (ii) $|H|=4$ and $|H / \beta| \in\{2,3\}$. In both cases $(H, 0)$ is a complete 1-hypergroup by Proposition 4 . The corresponding hyperproduct tables are derived from Remark 1.

Proposition 5. Let $(H, \circ)$ be a 1-hypergroup and let $a, b$ be elements of $H$ such that $\beta(a) \circ \beta(b)=\{1\}$ and $\beta(a) \circ \beta(a)=\beta(b)$. Then,

1. $\beta(b) \circ \beta(a)=\{1\}$ and $\beta(b) \circ \beta(b)=\beta(a)$;

2. if $a^{\prime}, a^{\prime \prime} \in \beta(a)$ and $a^{\prime} \circ a^{\prime \prime}=A$ then

(a) $A \circ x=x \circ A=\beta(a)$ for all $x \in \beta(b)$;

(b) if there exist $b^{\prime}, b^{\prime \prime} \in \beta(b)$ such that $b^{\prime} \circ b^{\prime \prime}=\left\{a^{\prime}\right\}$ or $b^{\prime} \circ b^{\prime \prime}=\left\{a^{\prime \prime}\right\}$ then $A=\beta(b)$. 
Proof. 1. The claim follows immediately from Theorem 1 .

2. (a) $\beta(a)=\beta\left(a^{\prime}\right)=a^{\prime} \circ 1=a^{\prime} \circ\left(a^{\prime \prime} \circ x\right)=\left(a^{\prime} \circ a^{\prime \prime}\right) \circ x=A \circ x$ and $\beta(a)=$ $\beta\left(a^{\prime \prime}\right)=1 \circ a^{\prime \prime}=\left(x \circ a^{\prime}\right) \circ a^{\prime \prime}=x \circ\left(a^{\prime} \circ a^{\prime \prime}\right)=x \circ A$.

(b) If $b^{\prime} \circ b^{\prime \prime}=\left\{a^{\prime}\right\}$, then $A=a^{\prime} \circ a^{\prime \prime}=\left(b^{\prime} \circ b^{\prime \prime}\right) \circ a^{\prime \prime}=b^{\prime} \circ\left(b^{\prime \prime} \circ a^{\prime \prime}\right)=b^{\prime} \circ 1=\beta(b)$. In the same way, if $b^{\prime} \circ b^{\prime \prime}=\left\{a^{\prime \prime}\right\}$ then $A=a^{\prime} \circ a^{\prime \prime}=a^{\prime} \circ\left(b^{\prime} \circ b^{\prime \prime}\right)=$ $\left(a^{\prime} \circ b^{\prime}\right) \circ b^{\prime \prime}=1 \circ b^{\prime \prime}=\beta(b)$.

In the forthcoming sections, we will determine the hyperproduct tables of 1-hypergroups of sizes 5 and 6 , up to isomorphisms. Since $\beta$ is an equivalence, the $\beta$-classes of a hypergroup $(H, \circ)$ determine a partition of $H$ in disjoint subsets. By Theorem $1(1)$, if $(H, \circ)$ is a finite 1-hypergroup such that $H=\{1,2, \ldots, n\}$ and $\omega_{H}=\{1\}$ then the first row and the first column of the hyperproduct table exhibits the sets of the partition. In order to find the 1-hypergroups of size $n$ with $|H / \beta|=r$, we will consider all the non-increasing partitions of the integer $(n-1)$ in exactly $(r-1)$ positive integers.

\section{1-Hypergroups of Size 5}

In this section we determine the hyperproduct tables of 1-hypergroups of size 5, apart of isomorphisms. Hence, we put $H=\{1,2,3,4,5\}$ and proceed with the analysis by considering the following cases, corresponding to the non-increasing partitions of 4 :

1. $|H / \beta|=2, \beta(2)=\{2,3,4,5\}$;

2. $|H / \beta|=3, \beta(2)=\{2,3,4\}, \beta(5)=\{5\}$;

3. $|H / \beta|=3, \beta(2)=\{2,3\}, \beta(4)=\{4,5\}$;

4. $|H / \beta|=4, \beta(2)=\{2,3\}, \beta(4)=\{4\}, \beta(5)=\{5\}$;

5. $|H / \beta|=5$ and $\beta(x)=\{x\}$ for all $x \in H$.

Case 1. In the first case $H / \beta \cong \mathbb{Z}_{2}$, so we only have the following complete hypergroup:

\begin{tabular}{cccccc}
\hline$\circ_{1}$ & 1 & 2 & 3 & 4 & 5 \\
\hline 1 & 1 & $2,3,4,5$ & $2,3,4,5$ & $2,3,4,5$ & $2,3,4,5$ \\
2 & $2,3,4,5$ & 1 & 1 & 1 & 1 \\
3 & $2,3,4,5$ & 1 & 1 & 1 & 1 \\
4 & $2,3,4,5$ & 1 & 1 & 1 & 1 \\
5 & $2,3,4,5$ & 1 & 1 & 1 & 1 \\
\hline
\end{tabular}

Case 2. By Proposition 4(2), $(H, O)$ is a complete hypergroup and so its hyperproduct table is the following, apart of isomorphisms:

\begin{tabular}{cccccc}
\hline$\circ_{2}$ & 1 & 2 & 3 & 4 & 5 \\
\hline 1 & 1 & $2,3,4$ & $2,3,4$ & $2,3,4$ & 5 \\
2 & $2,3,4$ & 5 & 5 & 5 & 1 \\
3 & $2,3,4$ & 5 & 5 & 5 & 1 \\
4 & $2,3,4$ & 5 & 5 & 5 & 1 \\
5 & 5 & 1 & 1 & 1 & $2,3,4$ \\
\hline
\end{tabular}


Case 3. Here $H / \beta \cong Z_{3}$ and, setting $\beta(2)=\{2,3\}$ and $\beta(4)=\{4,5\}$, we derive the following partial hyperproduct table:

\begin{tabular}{cccccc}
\hline$\circ$ & 1 & 2 & 3 & 4 & 5 \\
\hline 1 & 1 & 2,3 & 2,3 & 4,5 & 4,5 \\
2 & 2,3 & & & 1 & 1 \\
3 & 2,3 & & & 1 & 1 \\
4 & 4,5 & 1 & 1 & & \\
5 & 4,5 & 1 & 1 & & \\
\hline
\end{tabular}

By Proposition 5,

- $\quad$ if $a, b, a^{\prime}, b^{\prime}$ are elements in $\beta(2)$ then

$$
|a \circ b|=\left|a^{\prime} \circ b^{\prime}\right|=1 \quad \Longrightarrow \quad 4 \circ 4=4 \circ 5=5 \circ 4=5 \circ 5=\{2,3\} ;
$$

- if $a, b, a^{\prime}, b^{\prime}$ are elements in $\beta(4)$ then

$$
|a \circ b|=\left|a^{\prime} \circ b^{\prime}\right|=1 \quad \Longrightarrow \quad 2 \circ 2=2 \circ 3=3 \circ 2=3 \circ 3=\{4,5\} .
$$

Therefore, if we denote

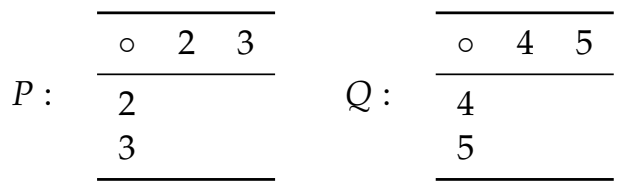

then we can restrict ourselves to the following three sub-cases:

- The tables $P$ and $Q$ do not contain any singleton entry. Here, one complete hypergroup arises,

\begin{tabular}{cccccc}
\hline$\circ_{3}$ & 1 & 2 & 3 & 4 & 5 \\
\hline 1 & 1 & 2,3 & 2,3 & 4,5 & 4,5 \\
2 & 2,3 & 4,5 & 4,5 & 1 & 1 \\
3 & 2,3 & 4,5 & 4,5 & 1 & 1 \\
4 & 4,5 & 1 & 1 & 2,3 & 2,3 \\
5 & 4,5 & 1 & 1 & 2,3 & 2,3 \\
\hline
\end{tabular}

- The table $P$ contains (one or more) singleton entries in the main diagonal only. Without loss of generality, we can set $2 \circ 2=\{4\}$ and obtain

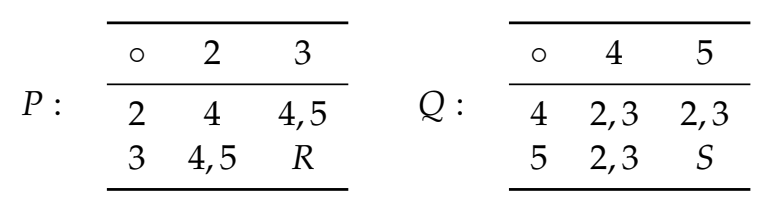

where $R \in\{\{4\},\{5\},\{4,5\}\}$ and $S \in\{\{3\},\{2,3\}\}$, that is to say there are 6 tables to examine. Rejecting the hyperproduct tables that are not reproducible and the isomorphic copies, we are left with the following 4 hypergroups:

\begin{tabular}{cccccc}
\hline$\circ_{4}$ & 1 & 2 & 3 & 4 & 5 \\
\hline 1 & 1 & 2,3 & 2,3 & 4,5 & 4,5 \\
2 & 2,3 & 4 & 4,5 & 1 & 1 \\
3 & 2,3 & 4,5 & 4,5 & 1 & 1 \\
4 & 4,5 & 1 & 1 & 2,3 & 2,3 \\
5 & 4,5 & 1 & 1 & 2,3 & 3 \\
\hline
\end{tabular}

\begin{tabular}{cccccc}
\hline$\circ_{5}$ & 1 & 2 & 3 & 4 & 5 \\
\hline 1 & 1 & 2,3 & 2,3 & 4,5 & 4,5 \\
2 & 2,3 & 4 & 4,5 & 1 & 1 \\
3 & 2,3 & 4,5 & 4,5 & 1 & 1 \\
4 & 4,5 & 1 & 1 & 2,3 & 2,3 \\
5 & 4,5 & 1 & 1 & 2,3 & 2,3 \\
\hline
\end{tabular}




\begin{tabular}{cccccc}
\hline$\circ_{6}$ & 1 & 2 & 3 & 4 & 5 \\
\hline 1 & 1 & 2,3 & 2,3 & 4,5 & 4,5 \\
2 & 2,3 & 4 & 4,5 & 1 & 1 \\
3 & 2,3 & 4,5 & 5 & 1 & 1 \\
4 & 4,5 & 1 & 1 & 2,3 & 2,3 \\
5 & 4,5 & 1 & 1 & 2,3 & 2,3 \\
\hline
\end{tabular}

\begin{tabular}{cccccc}
\hline$\circ_{7}$ & 1 & 2 & 3 & 4 & 5 \\
\hline 1 & 1 & 2,3 & 2,3 & 4,5 & 4,5 \\
2 & 2,3 & 4 & 4,5 & 1 & 1 \\
3 & 2,3 & 4,5 & 4 & 1 & 1 \\
4 & 4,5 & 1 & 1 & 2,3 & 2,3 \\
5 & 4,5 & 1 & 1 & 2,3 & 2,3 \\
\hline
\end{tabular}

- The table P contains at least one singleton entry off the main diagonal, for instance $2 \circ 3=\{4\}$. Consequently, from Proposition 5 we have

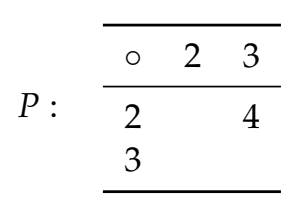$$
Q: \begin{array}{ccc}
\hline \circ & 4 & 5 \\
\cline { 2 - 3 } 4 & 2,3 & 2,3 \\
5 & 2,3 & 2,3 \\
\hline
\end{array}
$$

where every empty cell can be filled with $\{4\}$ or $\{5\}$ or $\{4,5\}$, giving rise to 27 more tables. After checking reproducibility and isomorphisms, we find the following 8 hypergroups:

\begin{tabular}{cccccc}
\hline$\circ_{8}$ & 1 & 2 & 3 & 4 & 5 \\
\hline 1 & 1 & 2,3 & 2,3 & 4,5 & 4,5 \\
2 & 2,3 & 4,5 & 4 & 1 & 1 \\
3 & 2,3 & 4,5 & 4,5 & 1 & 1 \\
4 & 4,5 & 1 & 1 & 2,3 & 2,3 \\
5 & 4,5 & 1 & 1 & 2,3 & 2,3 \\
\hline \multicolumn{7}{c}{} & & & \\
\hline$\circ_{10}$ & 1 & 2 & 3 & 4 & 5 \\
\hline 1 & 1 & 2,3 & 2,3 & 4,5 & 4,5 \\
2 & 2,3 & 4,5 & 4 & 1 & 1 \\
3 & 2,3 & 4,5 & 5 & 1 & 1 \\
4 & 4,5 & 1 & 1 & 2,3 & 2,3 \\
5 & 4,5 & 1 & 1 & 2,3 & 2,3 \\
\hline & & & & & \\
\hline$\circ_{12}$ & 1 & 2 & 3 & 4 & 5 \\
\hline 1 & 1 & 2,3 & 2,3 & 4,5 & 4,5 \\
2 & 2,3 & 4,5 & 4 & 1 & 1 \\
3 & 2,3 & 4 & 4,5 & 1 & 1 \\
4 & 4,5 & 1 & 1 & 2,3 & 2,3 \\
5 & 4,5 & 1 & 1 & 2,3 & 2,3 \\
\hline$\circ_{14}$ & 1 & 2 & 3 & 4 & 5 \\
\hline 1 & 1 & 2,3 & 2,3 & 4,5 & 4,5 \\
2 & 2,3 & 5 & 4 & 1 & 1 \\
3 & 2,3 & 4,5 & 5 & 1 & 1 \\
4 & 4,5 & 1 & 1 & 2,3 & 2,3 \\
5 & 4,5 & 1 & 1 & 2,3 & 2,3 \\
\hline & & & &
\end{tabular}

\begin{tabular}{cccccc}
\hline$\circ_{9}$ & 1 & 2 & 3 & 4 & 5 \\
\hline 1 & 1 & 2,3 & 2,3 & 4,5 & 4,5 \\
2 & 2,3 & 5 & 4 & 1 & 1 \\
3 & 2,3 & 4,5 & 4,5 & 1 & 1 \\
4 & 4,5 & 1 & 1 & 2,3 & 2,3 \\
5 & 4,5 & 1 & 1 & 2,3 & 2,3 \\
\hline
\end{tabular}

\begin{tabular}{cccccc}
\hline$\circ_{11}$ & 1 & 2 & 3 & 4 & 5 \\
\hline 1 & 1 & 2,3 & 2,3 & 4,5 & 4,5 \\
2 & 2,3 & 4,5 & 4 & 1 & 1 \\
3 & 2,3 & 5 & 4,5 & 1 & 1 \\
4 & 4,5 & 1 & 1 & 2,3 & 2,3 \\
5 & 4,5 & 1 & 1 & 2,3 & 2,3 \\
\hline$\circ_{13}$ & 1 & 2 & 3 & 4 & 5 \\
\hline 1 & 1 & 2,3 & 2,3 & 4,5 & 4,5 \\
2 & 2,3 & 4,5 & 4 & 1 & 1 \\
3 & 2,3 & 4 & 5 & 1 & 1 \\
4 & 4,5 & 1 & 1 & 2,3 & 2,3 \\
5 & 4,5 & 1 & 1 & 2,3 & 2,3 \\
\hline & & & & & \\
\hline$\circ_{15}$ & 1 & 2 & 3 & 4 & 5 \\
\hline 1 & 1 & 2,3 & 2,3 & 4,5 & 4,5 \\
2 & 2,3 & 5 & 4 & 1 & 1 \\
3 & 2,3 & 4 & 5 & 1 & 1 \\
4 & 4,5 & 1 & 1 & 2,3 & 2,3 \\
5 & 4,5 & 1 & 1 & 2,3 & 2,3 \\
\hline
\end{tabular}

Case 4. Here, being $|H / \beta|=4$, three more 1-hypergroups are obtained by considering that the quotient group $H / \beta$ is isomorphic to either the group $\mathbb{Z}_{4}$ or the group $\mathbb{Z}_{2} \times \mathbb{Z}_{2}$. 
- If $H / \beta \cong \mathbb{Z}_{4}$ and the $\beta$-class $\beta(2)$ is associated with a generator of $\mathbb{Z}_{4}$ then we have

\begin{tabular}{cccccc}
\hline$\circ_{16}$ & 1 & 2 & 3 & 4 & 5 \\
\hline 1 & 1 & 2,3, & 2,3 & 4 & 5 \\
2 & 2,3 & 4 & 4 & 5 & 1 \\
3 & 2,3 & 4 & 4 & 5 & 1 \\
4 & 4 & 5 & 5 & 1 & 2,3 \\
5 & 5 & 1 & 1 & 2,3 & 4 \\
\hline
\end{tabular}

- If $H / \beta \cong \mathbb{Z}_{4}$ and the $\beta$-class $\beta(2)$ is not associated with a generator of $\mathbb{Z}_{4}$ then we have

\begin{tabular}{cccccc}
\hline$\circ_{17}$ & 1 & 2 & 3 & 4 & 5 \\
\hline 1 & 1 & 2,3 & 2,3 & 4 & 5 \\
2 & 2,3 & 1 & 1 & 5 & 4 \\
3 & 2,3 & 1 & 1 & 5 & 4 \\
4 & 4 & 5 & 5 & 2,3 & 1 \\
5 & 5 & 4 & 4 & 1 & 2,3 \\
\hline
\end{tabular}

- $\quad$ If $H / \beta \cong \mathbb{Z}_{2} \times \mathbb{Z}_{2}$ then we have

\begin{tabular}{cccccc}
\hline$\circ_{18}$ & 1 & 2 & 3 & 4 & 5 \\
\hline 1 & 1 & 2,3 & 2,3 & 4 & 5 \\
2 & 2,3 & 1 & 1 & 5 & 4 \\
3 & 2,3 & 1 & 1 & 5 & 4 \\
4 & 4 & 5 & 5 & 1 & 2,3 \\
5 & 5 & 4 & 4 & 2,3 & 1 \\
\hline
\end{tabular}

Case 5. Lastly, in this case we have trivially $H \cong \mathbb{Z}_{5}$ as $|H / \beta|=5$.

Therefore we have obtained the following result.

Theorem 3. Apart of isomorphisms, there are 19 1-hypergroups of size 5. Of these hypergroups, exactly 7 are complete.

Remark 3. With the only exception of the hypergroup $\left(H, \mathrm{O}_{4}\right)$ in case 3 , the 1-hypergroups of size 5 can be determined by the construction defined in Section 3.1. In fact, the hypergroups $\left(H, \circ_{k}\right)$ with $k \in\{1,2,3\}$ are also complete. The hypergroups $\left(H, \circ_{k}\right)$ with $k \in\{5,6, \cdots, 15\}$ are obtained by considering $G \cong \mathbb{Z}_{3}, A_{1}=\{1\}, A_{2}=\{2,3\}, A_{3}=\{4,5\}$ and the functions $\varphi_{k}: A_{2} \times A_{2} \mapsto \mathcal{P}^{*}\left(A_{3}\right)$ defined as $\varphi_{k}(a, b)=a \circ_{k} b$ for $a, b \in A_{2}$ and $k \in\{5,6, \cdots, 15\}$.

\section{1-Hypergroups of Size 6}

In this section we classify the product tables of 1-hypergroups of size 6 , apart of isomorphisms. Hence, we assume $H=\{1,2,3,4,5,6\}, \omega_{H}=\{1\}$ and distinguish the following nine cases:

$$
\begin{array}{ll}
\text { 1. } & |H / \beta|=2, \beta(2)=\{2,3,4,5,6\} ; \\
\text { 2. } & |H / \beta|=3, \beta(2)=\{2,3,4,5\}, \beta(6)=\{6\} ; \\
\text { 3. } & |H / \beta|=3, \beta(2)=\{2,3,4\}, \beta(5)=\{5,6\} ; \\
\text { 4. } & |H / \beta|=4, H / \beta \cong \mathbb{Z}_{4}, \beta(2)=\{2,3,4\}, \beta(5)=\{5\}, \beta(6)=\{6\} ; \\
\text { 5. } & |H / \beta|=4, H / \beta \cong \mathbb{Z}_{4}, \beta(2)=\{2,3\}, \beta(4)=\{4,5\}, \beta(6)=\{6\} ; \\
\text { 6. } & |H / \beta|=4, H / \beta \cong \mathbb{Z}_{2} \times \mathbb{Z}_{2}, \beta(2)=\{2,3,4\}, \beta(5)=\{5\}, \beta(6)=\{6\} ; \\
\text { 7. } & |H / \beta|=4, H / \beta \cong \mathbb{Z}_{2} \times \mathbb{Z}_{2}, \beta(2)=\{2,3\}, \beta(4)=\{4,5\}, \beta(6)=\{6\} ; \\
\text { 8. } & |H / \beta|=5, \beta(2)=\{2,3\}, \beta(4)=\{4\}, \beta(5)=\{5\}, \beta(6)=\{6\} ; \\
\text { 9. } & |H / \beta|=6 .
\end{array}
$$


In all aforesaid cases, except case 3, we can give the hyperproduct tables of the 1-hypergroups, apart of isomorphisms. To achieve this goal, we use the partition of $H$ into $\beta$-classes, the involved quotient group and the reproducibility condition that the hyperproduct tables must satisfy. In case 3 , we obtain a too high number of tables and it is impossible to list them. Nevertheless, with the help of a computer algebra system, we are able to perform an exhaustive search of all possible hyperproduct tables and to determine their number, apart from isomorphisms. To improve readability, we postpone the discussion of case 3 at the end of this chapter.

Case 1. The quotient group $H / \beta$ is isomorphic to $\mathbb{Z}_{2}$.

\begin{tabular}{ccccccc}
\hline$\circ$ & 1 & 2 & 3 & 4 & 5 & 6 \\
\hline 1 & 1 & $2,3,4,5,6$ & $2,3,4,5,6$ & $2,3,4,5,6$ & $2,3,4,5,6$ & $2,3,4,5,6$ \\
2 & $2,3,4,5,6$ & 1 & 1 & 1 & 1 & 1 \\
3 & $2,3,4,5,6$ & 1 & 1 & 1 & 1 & 1 \\
4 & $2,3,4,5,6$ & 1 & 1 & 1 & 1 & 1 \\
5 & $2,3,4,5,6$ & 1 & 1 & 1 & 1 & 1 \\
6 & $2,3,4,5,6$ & 1 & 1 & 1 & 1 & 1 \\
\hline
\end{tabular}

Case 2. The quotient group $H / \beta$ is isomorphic to $\mathbb{Z}_{3}$.

\begin{tabular}{ccccccc}
\hline$\circ$ & 1 & 2 & 3 & 4 & 5 & 6 \\
\hline 1 & 1 & $2,3,4,5$ & $2,3,4,5$ & $2,3,4,5$ & $2,3,4,5$ & 6 \\
2 & $2,3,4,5$ & 6 & 6 & 6 & 6 & 1 \\
3 & $2,3,4,5$ & 6 & 6 & 6 & 6 & 1 \\
4 & $2,3,4,5$ & 6 & 6 & 6 & 6 & 1 \\
5 & $2,3,4,5$ & 6 & 6 & 6 & 6 & 1 \\
6 & 6 & 1 & 1 & 1 & 1 & $2,3,4,5$ \\
\hline
\end{tabular}

Case 4. By Corollary 1, we obtain two complete non-isomorphic hypergroups. In particular, where the only $\beta$-class of size larger than 1 is associated to a generator of $\mathbb{Z}_{4}$, we have the following hyperproduct table:

\begin{tabular}{ccccccc}
\hline$\circ$ & 1 & 2 & 3 & 4 & 5 & 6 \\
\hline 1 & 1 & $2,3,4$ & $2,3,4$ & $2,3,4$ & 5 & 6 \\
2 & $2,3,4$ & 5 & 5 & 5 & 6 & 1 \\
3 & $2,3,4$ & 5 & 5 & 5 & 6 & 1 \\
4 & $2,3,4$ & 5 & 5 & 5 & 6 & 1 \\
5 & 5 & 6 & 6 & 6 & 1 & $2,3,4$ \\
6 & 6 & 1 & 1 & 1 & $2,3,4$ & 5 \\
\hline
\end{tabular}

Instead, if the only $\beta$-class of size larger than 1 is associated to a non-generator of $\mathbb{Z}_{4}$, we obtain the following table:

\begin{tabular}{ccccccc}
\hline$\circ$ & 1 & 2 & 3 & 4 & 5 & 6 \\
\hline 1 & 1 & $2,3,4$ & $2,3,4$ & $2,3,4$ & 5 & 6 \\
2 & $2,3,4$ & 1 & 1 & 1 & 6 & 5 \\
3 & $2,3,4$ & 1 & 1 & 1 & 6 & 5 \\
4 & $2,3,4$ & 1 & 1 & 1 & 6 & 5 \\
5 & 5 & 6 & 6 & 6 & $2,3,4$ & 1 \\
6 & 6 & 5 & 5 & 5 & 1 & $2,3,4$ \\
\hline
\end{tabular}


Case 5. Considering that the group $\mathbb{Z}_{4}$ has only one element $x$ of order 2 and that $\beta(6)$ is the only $\beta$-class of size 1 , we have to examine two sub-cases, depending on whether the class $\beta(6)$ is associated to the element $x$ or not.

1. $|H / \beta|=4, H / \beta \cong \mathbb{Z}_{4}, \beta(2)=\{2,3\}, \beta(4)=\{4,5\}, \beta(6)=\{6\}$ and $\beta(6)$ associated to the only element of $\mathbb{Z}_{4}$ having order two;

2. $|H / \beta|=4, H / \beta \cong \mathbb{Z}_{4}, \beta(2)=\{2,3\}, \beta(4)=\{4,5\}, \beta(6)=\{6\}$ and $\beta(6)$ associated to a generator of $\mathbb{Z}_{4}$.

In the first case we obtain a complete hypergroup,

\begin{tabular}{ccccccc}
\hline$\circ$ & 1 & 2 & 3 & 4 & 5 & 6 \\
\hline 1 & 1 & 2,3 & 2,3 & 4,5 & 4,5 & 6 \\
2 & 2,3 & 6 & 6 & 1 & 1 & 4,5 \\
3 & 2,3 & 6 & 6 & 1 & 1 & 4,5 \\
4 & 4,5 & 1 & 1 & 6 & 6 & 2,3 \\
5 & 4,5 & 1 & 1 & 6 & 6 & 2,3 \\
6 & 6 & 4,5 & 4,5 & 2,3 & 2,3 & 1 \\
\hline
\end{tabular}

In the second case, by using the multiplicative table of $\mathbb{Z}_{4}$ and the reproducibility of $H$, we obtain the following partial table:

\begin{tabular}{ccccccc}
\hline$\circ$ & 1 & 2 & 3 & 4 & 5 & 6 \\
\hline 1 & 1 & 2,3 & 2,3 & 4,5 & 4,5 & 6 \\
2 & 2,3 & X & $Y$ & 6 & 6 & 1 \\
3 & 2,3 & $Z$ & $T$ & 6 & 6 & 1 \\
4 & 4,5 & 6 & 6 & 1 & 1 & 2,3 \\
5 & 4,5 & 6 & 6 & 1 & 1 & 2,3 \\
6 & 6 & 1 & 1 & 2,3 & 2,3 & 4,5 \\
\hline
\end{tabular}

with $X \cup Y=Z \cup T=X \cup Z=Y \cup T=\{4,5\}$. If we suppose that $X \in\{\{4\},\{4,5\}\}$, up to isomorphisms, we obtain 12 hyperproduct tables corresponding to the following values of the sets $X, Y, Z, T$ :

$\left(\star_{1}\right) X=\{4\}, Y=\{5\}, Z=\{5\}, T=\{4\}$;

$\left(\star_{2}\right) X=\{4\}, Y=\{5\}, Z=\{5\}, T=\{4,5\}$;

(*3) $X=\{4\}, Y=\{5\}, Z=\{4,5\}, T=\{4\}$;

( $\left.\star_{4}\right) \quad X=\{4\}, Y=\{5\}, Z=\{4,5\}, T=\{4,5\}$;

(*5) $X=\{4\}, Y=\{4,5\}, Z=\{5\}, T=\{4,5\}$;

(*6) $X=\{4\}, Y=\{4,5\}, Z=\{4,5\}, T=\{4\}$;

(*7) $X=\{4\}, Y=\{4,5\}, Z=\{4,5\}, T=\{5\}$;

$\left(\star_{8}\right) \quad X=\{4\}, Y=\{4,5\}, Z=\{4,5\}, T=\{4,5\}$;

(*9) $X=\{4,5\}, Y=\{4\}, Z=\{4\}, T=\{4,5\}$;

$\left(\star_{10}\right) X=\{4,5\}, Y=\{4\}, Z=\{5\}, T=\{4,5\}$;

$\left(\star_{11}\right) X=\{4,5\}, Y=\{4\}, Z=\{4,5\}, T=\{4,5\}$;

$\left(\star_{12}\right) X=\{4,5\}, Y=\{4,5\}, Z=\{4,5\}, T=\{4,5\}$.

Remark 4. The previous 12 hypergroups can be derived from the construction shown in Section 3.1, where we let $G \cong \mathbb{Z}_{4}, A_{1}=\{1\}, A_{2}=\{2,3\}, A_{3}=\{4,5\}, A_{4}=\{6\}$, and $\varphi: A_{2} \times$ $A_{2} \mapsto \mathcal{P}^{*}\left(A_{3}\right)$ is the function defined as $\varphi(a, b)=a \star_{k} b$ for $a, b \in A_{2}$ and $k \in\{1,2, \ldots, 12\}$. Incidentally, we note that the hypergroup arising from $\star_{12}$ is also complete. 
Case 6. In this case we obtain only one 1-hypergroup, which is also complete:

\begin{tabular}{ccccccc}
\hline$\circ$ & 1 & 2 & 3 & 4 & 5 & 6 \\
\hline 1 & 1 & $2,3,4$ & $2,3,4$ & $2,3,4$ & 5 & 6 \\
2 & $2,3,4$ & 1 & 1 & 1 & 6 & 5 \\
3 & $2,3,4$ & 1 & 1 & 1 & 6 & 5 \\
4 & $2,3,4$ & 1 & 1 & 1 & 6 & 5 \\
5 & 5 & 6 & 6 & 6 & 1 & $2,3,4$ \\
6 & 6 & 5 & 5 & 5 & $2,3,4$ & 1 \\
\hline
\end{tabular}

Case 7. In this case, we also obtain only one 1-hypergroup, which is also complete:

\begin{tabular}{ccccccc}
\hline & 1 & 2 & 3 & 4 & 5 & 6 \\
\hline 1 & 1 & 2,3 & 2,3 & 4,5 & 4,5 & 6 \\
2 & 2,3 & 1 & 1 & 6 & 6 & 4,5 \\
3 & 2,3 & 1 & 1 & 6 & 6 & 4,5 \\
4 & 4,5 & 6 & 6 & 1 & 1 & 2,3 \\
5 & 4,5 & 6 & 6 & 1 & 1 & 2,3 \\
6 & 6 & 4,5 & 4,5 & 2,3 & 2,3 & 1 \\
\hline
\end{tabular}

Case 8. Here the quotient group is isomorphic to $Z_{5}$ and we deduce one complete hypergroup:

\begin{tabular}{ccccccc}
\hline & 1 & 2 & 3 & 4 & 5 & 6 \\
\hline 1 & 1 & 2,3 & 2,3 & 4 & 5 & 6 \\
2 & 2,3 & 4 & 4 & 5 & 6 & 1 \\
3 & 2,3 & 4 & 4 & 5 & 6 & 1 \\
4 & 4 & 5 & 5 & 6 & 1 & 2,3 \\
5 & 5 & 6 & 6 & 1 & 2,3 & 4 \\
6 & 6 & 1 & 1 & 2,3 & 4 & 5 \\
\hline
\end{tabular}

Case 9. Here $\beta(x)=\{x\}, \forall x \in\{2,3,4,5,6\}$, and so $H$ is a group of order 6 , that is $H \cong \mathbb{Z}_{6}$ or $H \cong S_{3}$.

To conclude the review of 1-hypergroups of size 6, hereafter we consider the most challenging case, where a very high number of tables arises.

Case 3. Here the quotient group $H / \beta$ is isomorphic to $\mathbb{Z}_{3}, \beta(2)=\{2,3,4\}$ and $\beta(5)=\{5,6\}$. In this case there is only one complete 1-hypergroup; its multiplicative table is the following:

\begin{tabular}{ccccccc}
\hline$\circ$ & 1 & 2 & 3 & 4 & 5 & 6 \\
\hline 1 & 1 & $2,3,4$ & $2,3,4$ & $2,3,4$ & 5,6 & 5,6 \\
2 & $2,3,4$ & 5,6 & 5,6 & 5,6 & 1 & 1 \\
3 & $2,3,4$ & 5,6 & 5,6 & 5,6 & 1 & 1 \\
4 & $2,3,4$ & 5,6 & 5,6 & 5,6 & 1 & 1 \\
5 & 5,6 & 1 & 1 & 1 & $2,3,4$ & $2,3,4$ \\
6 & 5,6 & 1 & 1 & 1 & $2,3,4$ & $2,3,4$ \\
\hline
\end{tabular}

In order to find the other 1-hypergroups, we make sure that the sub-cases we are dealing with are disjoint from each other, which means that a hypergroup of a sub-case can not be isomorphic to a hypergroup of another sub-case. 
If $(H, \circ)$ is not a complete hypergroup then we can start from the partial table

\begin{tabular}{ccccccc}
\hline$\circ$ & 1 & 2 & 3 & 4 & 5 & 6 \\
\hline 1 & 1 & $2,3,4$ & $2,3,4$ & $2,3,4$ & 5,6 & 5,6 \\
2 & $2,3,4$ & & & & 1 & 1 \\
3 & $2,3,4$ & & & & 1 & 1 \\
4 & $2,3,4$ & & & & 1 & 1 \\
5 & 5,6 & 1 & 1 & 1 & & \\
6 & 5,6 & 1 & 1 & 1 & & \\
\hline
\end{tabular}

and the partial sub-tables

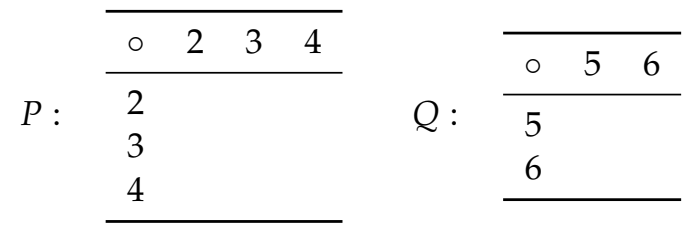

Taking into account Proposition 5, there are three options:

1. In the partial table $Q$ there is at least one hyperproduct which is a singleton, for instance $\{2\}$, and for all $a, a^{\prime} \in\{2,3,4\}$ we have $a \circ a^{\prime}=\{5,6\}$. We consider two sub-cases:

(1a) the singleton can appear only in the main diagonal:

$$
\begin{aligned}
& Q: \begin{array}{lll}
\hline \circ & 5 & 6 \\
\hline 5 & 2 & R
\end{array} \\
& \begin{array}{lll}
6 & S & T
\end{array}
\end{aligned}
$$

By reproducibility, we have $R, S \in\{\{3,4\},\{2,3,4\}\}$ and $T \in \mathcal{P}^{*}(\{2,3,4\})$. This yields $2^{2} \cdot 7=28$ tables to examine.

(1b) The singleton must appear off the main diagonal,

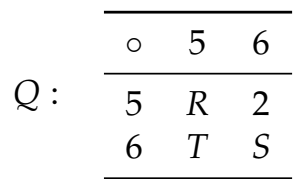

with $R, S \in\{\{3,4\},\{2,3,4\}\}$ and $T \in \mathcal{P}^{*}(\{2,3,4\})$. Thus other $2^{2} \cdot 7=28$ tables arise.

2. The partial table $Q$ contains at least one hyperproduct of size two, for instance $\{2,3\}$, but there are no singletons inside $Q$. Moreover, for all $a, a^{\prime} \in\{2,3,4\}$, we have $a \circ a^{\prime}=\{5,6\}$. We obtain two subcases, again:

(2a) the hyperproduct $\{2,3\}$ can appear only in the main diagonal,

$$
Q: \begin{array}{ccc}
\hline \circ & 5 & 6 \\
\cline { 2 - 3 } & 2,3 & 2,3,4 \\
6 & 2,3,4 & \\
\hline
\end{array}
$$

and $6 \circ 6 \in\{\{2,3\},\{2,4\},\{3,4\},\{2,3,4\}\}$. Hence, 4 cases tables arise. 
(2b) the hyperproduct $\{2,3\}$ must appear out of the main diagonal,

$$
Q: \begin{array}{ccc}
\hline \circ & 5 & 6 \\
\hline 5 & R & 2,3 \\
6 & S & T \\
\hline
\end{array}
$$

the hyperproducts $R$ and $T$ belong to the set $\{\{2,4\},\{3,4\},\{2,3,4\}\}$ and $S \in\{\{2,3\},\{2,4\},\{3,4\},\{2,3,4\}\}$. Therefore $3^{2} \cdot 4=36$ cases arise.

3. The partial table $P$ contains at least one singleton. Without loss in generality, we can suppose that $\{5\}$ is among them. From Proposition 5 we deduce $5 \circ 5=5 \circ 6=$ $6 \circ 5=\{2,3,4\}$. The following two possibilities arise:

(3a) Singletons can appear only in the main diagonal of $P$. Therefore we put $2 \circ 2=\{5\}$ and obtain

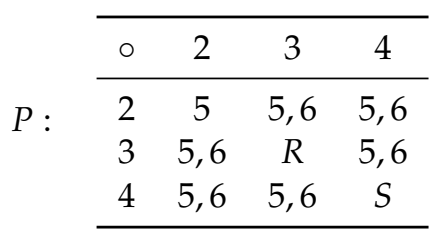

$Q:$\begin{tabular}{ccc}
\hline$\circ$ & 5 & 6 \\
\hline 5 & $2,3,4$ & $2,3,4$ \\
6 & $2,3,4$ & $T$ \\
\hline
\end{tabular}

where $R, S \in\{\{5\},\{6\},\{5,6\}\}$. Moreover, from Proposition 5, we deduce that $T \neq\{2\}$, that is $T \in \mathcal{P}^{*}(\{2,3,4\})-\{\{2\}\}$, and $3^{2} \cdot 6=54$ cases arise.

(3b) There is a singleton cell off the main diagonal of $P$, for instance, $2 \circ 3=\{5\}$. We obtain

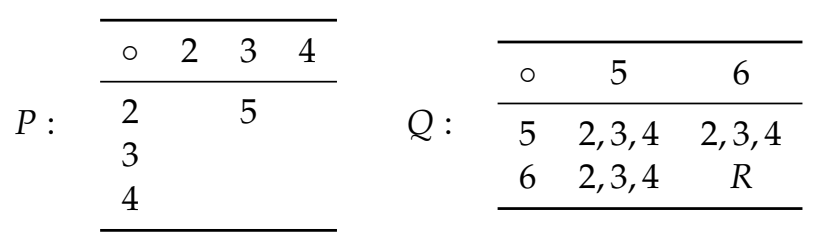

We consider two sub-cases:

i. $\quad R=\{2,3,4\}$ : the 8 empty cells in table $P$ can be filled with either $\{5\}$, or $\{6\}$, or $\{5,6\}$. Hence, $3^{8}$ cases arise.

ii. $|R|<3$ : from Proposition $5, R \neq\{2\}, R \neq\{3\}$, and so $R \in\{\{4\},\{2,3\}$, $\{2,4\},\{3,4\}\}$. Moreover the table $P$ can not contain the hyperproduct $\{6\}$, that is every cell in $P$ has to be filled with $\{5\}$ or $\{5,6\}$. Thus, $2^{8} \cdot 4=512$ cases arise.

All the previous sub-cases have been examined with the help of a computer algebra system based on MATLAB R2018a running on an iMac 2009 with an Intel Core 2 processor (3.06 GHz, 4 GB RAM). The complete enumeration of all 1-hypergroups in case 3 took about 2 min utilizing the subdivision into sub-cases described above, while without that subdivision the running time for solving case 3 exceeded $90 \mathrm{~min}$. We report in Table 1 the number of 1-hypergroups found in each sub-case considered above, up to isomorphisms.

Table 1. Number of non-isomorphic, non-complete 1-hypergroups found in case $3,|H|=6$.

\begin{tabular}{cccccccc}
\hline Case & $\mathbf{( 1 a )}$ & $\mathbf{( 1 b )}$ & $\mathbf{( 2 a )}$ & $\mathbf{( 2 b )}$ & $\mathbf{( 3 a})$ & $\mathbf{( 3 b )}$ & Total \\
\hline Hypergroups & 13 & 13 & 3 & 12 & 12 & 1180 & 1233 \\
\hline
\end{tabular}

Remark 5. The 1-hypergroups in sub-cases (1a), (1b), (2a) and (2b) can be derived from the construction shown in Section 3.1, where $G \cong \mathbb{Z}_{3}, A_{1}=\{1\}, A_{2}=\{5,6\}, A_{3}=\{2,3,4\}$ and $\varphi: A_{2} \times A_{2} \mapsto \mathcal{P}^{*}\left(A_{3}\right)$ is the function defined by the corresponding partial tables $Q$. 
In Table 2 we summarize the results obtained in our case-by-case review of 1-hypergroups of order 6 . In that table, we report the number of 1-hypergroups found in each case and the number of complete hypergroups among them. Theorem 4 states the conclusion.

Table 2. Number of non-isomorphic 1-hypergroups, $|H|=6$.

\begin{tabular}{ccccccccccc}
\hline Case & $\mathbf{1}$ & $\mathbf{2}$ & $\mathbf{3}$ & $\mathbf{4}$ & $\mathbf{5}$ & $\mathbf{6}$ & $\mathbf{7}$ & $\mathbf{8}$ & $\mathbf{9}$ & Total \\
\hline Hypergroups & 1 & 1 & 1234 & 2 & 13 & 1 & 1 & 1 & 2 & 1256 \\
Complete & 1 & 1 & 1 & 2 & 2 & 1 & 1 & 1 & 2 & 11 \\
\hline
\end{tabular}

Theorem 4. Up to isomorphisms, there are 1256 1-hypergroups of size 6, of which 11 are complete.

\section{Conclusions and Directions for Further Research}

A 1-hypergroup is a hypergroup $(H, \circ)$ where the kernel of the canonical projection $\varphi: H \mapsto H / \beta$ is a singleton. In this paper, we enumerate the 1 -hypergroups of size 5 and 6. The main results are given in Theorem 3 for $|H|=5$ and Theorem 4 for $|H|=6$. In particular, in Section 4 we show a representation of the 19 1-hypergroups of size 5 . To achieve this goal, we exploit the partition of $H$ induced by $\beta$. In this way, we reduce the analysis of a tough problem to that of a few sub-problems that can be solved explicitly or by means of scientific computing software on an ordinary desktop computer. Moreover, in Section 3.1 we give a construction of hypergroups which, under certain conditions, are 1-hypergroups. That construction is very flexible and many 1-hypergroups of size 5 and 6 can be determined in that way.

To highlight a direction for possible further research, we point out that many hypergroups found in the present work are also join spaces or transposition hypergroups. To be precise, let $(H, \circ)$ be a hypergroup and, for every $a, b \in H$, let $a / b$ and $b \backslash a$ denote the sets $\{x \in H \mid a \in x \circ b\}$ and $\{x \in H \mid a \in b \circ x\}$, respectively. The commutative hypergroups fulfilling the transposition axiom, that is

$$
a / b \cap c / d \neq \varnothing \Longrightarrow a \circ d \cap b \circ c \neq \varnothing
$$

for all $a, b, c, d \in H$ are called join spaces. These hypergroups have been widely used in Geometry [31,32]. In [33] Jantosciak generalized the transposition axiom to arbitrary hypergroups as follows:

$$
b \backslash a \cap c / d \neq \varnothing \Longrightarrow a \circ d \cap b \circ c \neq \varnothing,
$$

for all $a, b, c, d \in H$. These particular hypergroups are called transposition hypergroups. A number of results on transposition hypergroups can be found in, e.g., [33-35]. For example, it is known that the complete hypergroups are also transposition hypergroups. The construction shown in Section 3.1 produces transposition hypergroups when $a \star d \cap b \star$ $c \neq \varnothing$, for all $a, b \in A_{i}$ and $c, d \in A_{j}$. Indeed, if $x \in b \backslash a \cap c / d$ then $a \in b \circ x$ and $c \in x \circ d$. Thus, we have $a \circ d \cup b \circ c \subseteq b \circ x \circ d$. By point 3. of Proposition 1, there exists $k \in G$ such that $b \circ x \circ d=A_{k}$. By definition of $\circ$, if $k \neq i j$ then $a \circ d=b \circ c=A_{k}$. Otherwise, if $k=i j$ then we have $a, b \in A_{i}, c, d \in A_{j}, a \circ d=a \star d$ and $b \circ c=b \star c$. Hence, by hypotesis, $a \circ d \cap b \circ c \neq \varnothing$.

Based on the preceding comment, we plan to characterize and enumerate the 1-hypergroups of small size that also are join spaces or transposition hypergroups in further works.

Author Contributions: Conceptualization, investigation, writing-original draft: M.D.S., D.F. (Domenico Freni), and G.L.F.; software, writing-review and editing: D.F. (Dario Fasino). All authors have read and agreed to the published version of the manuscript. 
Funding: The research work of Mario De Salvo was funded by Università di Messina, Italy, grant FFABR Unime 2019. Giovanni Lo Faro was supported by INdAM-GNSAGA, Italy, and by Università di Messina, Italy, grant FFABR Unime 2020. The work of Dario Fasino was partially supported by INdAM-GNCS, Italy.

Conflicts of Interest: The authors declare no conflict of interest.

\section{References}

1. Massouros, G.; Massouros, C. Hypercompositional algebra, computer science and geometry. Mathematics 2020, 8, 1338. [CrossRef]

2. Koskas, H. Groupoïdes, demi-hypergroupes et hypergroupes. J. Math. Pures Appl. 1970, 49, 155-192.

3. Vougiouklis, T. Fundamental relations in hyperstructures. Bull. Greek Math. Soc. 1999, 42, 113-118.

4. Corsini, P. Prolegomena of Hypergroup Theory; Aviani Editore: Tricesimo, Italy, 1993.

5. Davvaz, B.; Salasi, A. A realization of hyperrings. Commun. Algebra 2006, 34, 4389-4400. [CrossRef]

6. De Salvo, M.; Lo Faro, G. On the $n *$-complete hypergroups. Discret. Math. 1999, 208/209, 177-188. [CrossRef]

7. De Salvo, M.; Fasino, D.; Freni, D.; Lo Faro, G. Semihypergroups obtained by merging of 0-semigroups with groups. Filomat 2018, 32, 4177-4194.

8. Freni, D. Une note sur le cœur d'un hypergroup et sur la clôture transitive $\beta^{*}$ de $\beta$. Riv. Mat. Pura Appl. 1991, 8, $153-156$.

9. Freni, D. Strongly transitive geometric spaces: Applications to hypergroups and semigroups theory. Commun. Algebra 2004, 32, 969-988. [CrossRef]

10. Gutan, M. On the transitivity of the relation $\beta$ in semihypergroups. Rend. Circ. Mat. Palermo 1996, 45, 189-200. [CrossRef]

11. Norouzi, M.; Cristea, I. Fundamental relation on $m$-idempotent hyperrings. Open Math. 2017, 15, 1558-1567. [CrossRef]

12. De Salvo, M.; Fasino, D.; Freni, D.; Lo Faro, G. On hypergroups with a $\beta$-class of finite height. Symmetry 2020, 12, 168. [CrossRef]

13. Sadrabadi, E.; Davvaz, B. Atanassov's intuitionistic fuzzy grade of a class of non-complete 1-hypergroups. J. Intell. Fuzzy Syst. 2014, 26, 2427-2436. [CrossRef]

14. Fasino, D.; Freni, D. Fundamental relations in simple and 0-simple semi-hypergroups of small size. Arab. J. Math. 2012, 1, 175-190. [CrossRef]

15. Freni, D. Minimal order semi-hypergroups of type $U$ on the right. II. J. Algebra 2011, 340, 77-89. [CrossRef]

16. Heidari, D.; Freni, D. On further properties of minimal size in hypergroups of type $U$ on the right. Comm. Algebra 2020, 48 , 4132-4141. [CrossRef]

17. Fasino, D.; Freni, D. Existence of proper semi-hypergroups of type $U$ on the right. Discrete Math. 2007, 307, 2826-2836. [CrossRef]

18. De Salvo, M.; Fasino, D.; Freni, D.; Lo Faro, G. Fully simple semihypergroups, transitive digraphs, and Sequence A000712. J. Algebra 2014, 415, 65-87. [CrossRef]

19. De Salvo, M.; Freni, D.; Lo Faro, G. Fully simple semihypergroups. J. Algebra 2014, 399, 358-377. [CrossRef]

20. De Salvo, M.; Freni, D.; Lo Faro, G. Hypercyclic subhypergroups of finite fully simple semihypergroups. J. Mult. Valued Log. Soft Comput. 2017, 29, 595-617.

21. De Salvo, M.; Fasino, D.; Freni, D.; Lo Faro, G. A family of 0-simple semihypergroups related to sequence A00070. J. Mult. Valued Log. Soft Comput. 2016, 27, 553-572.

22. De Salvo, M.; Freni, D.; Lo Faro, G. On hypercyclic fully zero-simple semihypergroups. Turk. J. Math. 2019, 43, 1905-1918. [CrossRef]

23. De Salvo, M.; Freni, D.; Lo Faro, G. On further properties of fully zero-simple semihypergroups. Mediterr. J. Math. 2019, 16, 48. [CrossRef]

24. Sonea, A.; Cristea, I. The class equation and the commutativity degree for complete hypergroups. Mathematics $2020,8,2253$. [CrossRef]

25. Antampoufis, N.; Spartalis, S.; Vougiouklis, T. Fundamental relations in special extensions. In Algebraic Hyperstructures and Applications; Vougiouklis, T., Ed.; Spanidis Press: Xanthi, Greece, 2003; pp. 81-89.

26. Davvaz, B. Semihypergroup Theory; Academic Press: London, UK, 2016.

27. Davvaz, B.; Leoreanu-Fotea, V. Hyperring Theory and Applications; International Academic Press: Palm Harbor, FL, USA, 2007.

28. De Salvo, M.; Lo Faro, G. A new class of hypergroupoids associated with binary relations. J. Mult. Valued Log. Soft Comput. 2003, 9, 361-375.

29. Massouros, G.G. The subhypergroups of the fortified join hypergroup. Ital. J. Pure Appl. Math. 1997, 2, 51-63.

30. Naz, S.; Shabir, M. On soft semihypergroups. J. Intell. Fuzzy Syst. 2014, 26, 2203-2213. [CrossRef]

31. Prenowitz, W. A contemporary approach to classical geometry. Am. Math. Month. 1961, 68, 1-67. [CrossRef]

32. Prenowitz, W.; Jantosciak, J. Join Geometries. A Theory of Convex Sets and Linear Geometry; Springer: New York, NY, USA, 1979.

33. Jantosciak, J. Transposition hypergroups: Noncommutative join spaces. J. Algebra 1997, 187, 97-119. [CrossRef]

34. Jantosciak, J.; Massouros, C.G. Strong identities and fortification in transposition hypergroups. J. Discrete Math. Sci. Cryptogr. 2003, 6, 169-193. [CrossRef]

35. Massouros, C.G.; Massouros, G.G. The transposition axiom in hypercompositional structures. Ratio Math. 2011, 21 , 75-90. 